\title{
Ablation of nanoparticles and efficient harmonic generation using a 1-kHz laser
}

\author{
R. A. Ganeev, ${ }^{1,2}$ C. Hutchison, ${ }^{1}$ I. Lopez-Quintas, ${ }^{3}$ F. McGrath, ${ }^{1}$ D. Y. Lei,,${ }^{1,4}$ M. Castillejo, ${ }^{3}$ and J. P. Marangos ${ }^{1}$ \\ ${ }^{1}$ Blackett Laboratory, Imperial College London, Prince Consort Road, London SW7 2AZ, United Kingdom \\ ${ }^{2}$ Voronezh State University, Voronezh 394006, Russia \\ ${ }^{3}$ Instituto de Química Física Rocasolano, CSIC, Serrano 119, 28006 Madrid, Spain \\ ${ }^{4}$ Department of Applied Physics, The Hong Kong Polytechnic University, Hong Kong, China
}

(Received 22 March 2013; published 3 September 2013)

\begin{abstract}
We report on high-order-harmonic generation in nanoparticle-containing laser ablation plasmas using a Ti:sapphire driving laser at a repetition rate of $1 \mathrm{kHz}$ and on the relation of the harmonic signals with the morphological and spectroscopic characteristics of the ablation deposits. The study of the deposits has allowed the definition of ablation conditions, leading to the generation of a plasma containing nanoparticles of similar sizes to the ones used as target material. The corresponding plasma plumes lead to efficient conversion of the IR photons towards the short-wavelength range through high-order harmonic generation and show superior performance to those corresponding to bulk targets of the same metallic element.
\end{abstract}

DOI: 10.1103/PhysRevA.88.033803

PACS number(s): 42.65.Ky

\section{INTRODUCTION}

Methods for improving the notoriously low efficiency of frequency conversion of coherent photons from commonly used laser sources towards the short-wavelength range include those related with monitoring the processes involved at the microscopic level and those related with macroscopic effects of the laser-matter interaction. Studies of high-order-harmonic generation (HHG), as a most commonly used process for frequency up-conversion of laser radiation from the infrared to the extreme ultraviolet, have incorporated various procedures for improving the conversion efficiency. Gases and surfaces, and more recently laser plasmas, are the nonlinear media subjected to these investigations. Initially, the reported frequency conversion efficiency $(\eta)$ in these media did not exceed $10^{-8}-10^{-7}$, values which are absolutely insufficient for either scientific studies or for practical application of these xuv sources. Investigations of HHG from gaseous media (initiated in 1987 [1-3]) and from laser plasmas (initiated in 1992 [4]) are connected with the search of advanced methods for improvement of the harmonic conversion efficiency. Nowadays, the quest for new methods remains as one of the main goals of nonlinear optics.

The very low values of $\eta$ reported for HHG are related with few peculiarities of the frequency up-conversion process in isotropic low-density media. For low-order harmonic generation (HG) in condensed media (i.e., nonlinear crystals), the initially low values of $\eta$ [5] were considerably improved (up to $10^{-1}-6 \times 10^{-1}$ ) thanks to the development of the phase-matching technique, which facilitates the participation of large amount of emitters, at typical densities of $10^{23}-10^{24} \mathrm{~cm}^{-3}$, involving the whole length of the nonlinear condensed medium. For generation of coherent radiation in a shorter wavelength region via frequency up-conversion, other nonlinear media had to be sought to avoid the considerable absorption during propagation of converted photons. Gases and, more recently, laser-generated plasmas have replaced condensed materials as media for HG of short-wavelength radiation. However, in these cases, the low concentrations of emitters $\left(\sim 10^{17}-10^{18} \mathrm{~cm}^{-3}\right)$ results in considerably low $\eta$ values for $\mathrm{HHG}$, due to the quadratic dependence on density
[1]. To amend the low HHG conversion efficiencies, various approaches have been proposed based on improving the influence of macroscopic propagation effects on the process of wavelength conversion. Those include optimization of the Gouy phase, addition to the nonlinear medium of a species with positive or negative dispersion for achieving phase matching, application of quasi-phase-matching conditions using few gas jets, laser beam aperturing, etc. ([2,6-10]; also see monograph [11] and references therein).

Another strategy to improve the HG conversion efficiency involves consideration of the microscopic processes, e.g., those related with the response of single atoms/ions instead of considering the nonlinear medium as an ensemble. To that purpose, the involvement of resonance processes in the enhancement of the nonlinear optical response of the medium has been considered. This was demonstrated for low-order HG in the vacuum ultraviolet (80-100 nm [11]). However, the use of a limited number of gaseous media did not allow the application of this effect to HHG in the xuv range. Alternatively, it was found that laser-generated plasmas were exceptionally suitable media in the search for resonance effects that could enhance the nonlinear response for a single harmonic in the short-wavelength range. One example is the laser-generated plasma from indium, where resonant effects lead to conversion efficiencies for HHG of around $10^{-4}$ for a single (13th) harmonic [12]. This value is 1 order of magnitude higher than that measured in gases [10] and plasmas [13] using various of the above-mentioned approaches related with improving the contribution of macroscopic processes in conditions far from resonance.

The use of clusters as nonlinear media is a scheme that has also been explored to increase the values of $\eta$ in HHG. The first attempts in this direction were made using gaseous clusters [14-19]. In this case, the improvement of $\eta$ is not only related with the increase of concentration of emitters, but also with specific properties of clusters. The cross section of recombination of the accelerated electron with the parent ion is higher for clusters than for atoms [20]. Also investigated are the effect on HG efficiency of the local field enhancement in the cluster vicinity, and of the plasmonic properties of 
nanoparticles and nanoantennas in gaseous media [21-24]. In particular, the increase of HHG generation efficiency due to surface plasmon resonance (SPR) effects was demonstrated in fullerene media [25,26]. Recently, due to the wide availability of nanoparticles in the market, it has become possible to generate by laser ablation a plasma containing a large amount of nanoparticles, and the enhancement of HHG in nanoparticle plasmas has been confirmed in various laboratories ( [27-29], see also the review of those studies [30]). The reported improvement in $\eta$ using various metal clusters showed the appeal of this approach.

However, former HHG studies in nanoparticle laser plasmas were only carried out using relatively low-repetition-rate lasers $(10 \mathrm{~Hz})$ due to the difficulty of maintaining a stable nanoparticle-containing plasma plume at high pulse repetition rates. As the harmonic average power increases with the repetition rate, it is highly desirable to maintain the concentration of nanoparticles in the plasma upon repetitive irradiation of the cluster-containing ablation target. A search for methods to ensure stable cluster concentration in the plasma will facilitate the increase, not only of the values of $\eta$ in HHG, but also of the average power of generated harmonics. At the same time, stable nanoparticle plasma conditions allow the investigation of the effect of various cluster properties, particularly SPR, in HG.

In this work, we demonstrate a method for stabilization of laser plasma plumes containing metal ( $\mathrm{Ag}, \mathrm{Al}$, and $\mathrm{Cu}$ ) nanoparticles, analyze the morphology and absorption spectra of the ablation deposits, and show the advantages of using a nanoparticle-containing plasma for HHG at a high pulse repetition rate $(1 \mathrm{kHz})$. Analysis of the properties of the ablation materials deposited on a substrate has allowed defining the conditions to generate plasmas, which contain nanoparticles of the same characteristics as the ones used as target material. Comparison with the harmonics generated in the ablation plasmas of bulk targets of the same metallic element clearly demonstrates the superior efficiency of the nanoparticle-based targets. We also discuss the mechanisms of efficient $\mathrm{HG}$ in cluster-containing media.

\section{EXPERIMENTAL PROCEDURE}

A 1-kHz chirped pulse amplification (CPA) Ti:sapphire laser (Red Dragon, KML, Inc.) was used as the main laserdriving source. Part of the uncompressed CPA radiation was split off from the beam line prior to the compressor stage (780 nm, $1.3 \mathrm{~mJ}, 160 \mathrm{ps)}$ and used for ablation of the targets placed in a vacuum chamber (Fig. 1). This beam was loosely focused onto the target with a spot size of about $500 \mu \mathrm{m}$. The resulting intensity on the target surface, in the range of $6 \times 10^{9}-2 \times 10^{10} \mathrm{~W} \mathrm{~cm}^{-2}$, created a weakly ionized plasma plume. After a delay of $\sim 40 \mathrm{~ns}$, required for formation and expansion of the plume away from the surface of the target, the compressed laser pulse $(780 \mathrm{~nm}, 1 \mathrm{~mJ}, 30 \mathrm{fs})$ was focused onto the plasma using a 200-mm focal length mirror at an intensity of $4 \times 10^{14} \mathrm{~W} \mathrm{~cm}^{-2}$ to generate the high-order harmonics. The distance between the target and the fundamental beam axis was maintained at $\sim 100 \mu \mathrm{m}$. The position of the focus relative to the plume was chosen to maximize HG efficiency. The harmonic emission was analyzed using an xuv spectrometer

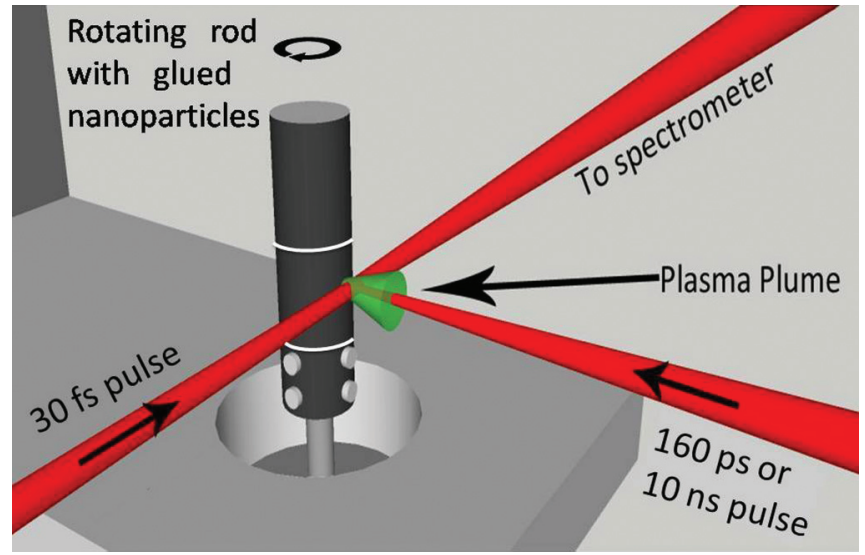

FIG. 1. (Color online) Experimental setup for ablation of a rotating rod coated by a layer of metallic nanoparticles with further harmonic generation in the plasma plume. The nanoparticles were glued on the surface of the rod on the area limited by the two curved white lines.

consisting of a flat field grating and a microchannel plate coupled to a phosphor screen. Images of harmonics were captured using a CCD camera.

Nanoparticle powders were purchased from US Research Nanomaterials, Inc. Small-sized (20-25 nm) Ag, Al, and $\mathrm{Cu}$ nanoparticles were used in most of the experiments, although we also investigated large (100-150 nm) Ag and Al nanoparticles. We aimed at comparing the characteristic morphology and size of the initial nanoparticles constituting the ablation target material with those of the deposits collected on silicon wafers placed at a distance of $4 \mathrm{~cm}$. The range of sizes also provided the case for studying the dependence of the nonlinear optical response of the corresponding laser plasmas on this parameter. The nanoparticle powders were prepared as targets by mixing with cyanoacrylate-based glue and coating the surface of a rotating rod with the mixture. The coated layer was further polished to offer a uniform surface to laser irradiation. To prevent overheating and damage of the same spot of the target from repeated laser ablation pulses, the rods were rotated at a speed of $15 \mathrm{rpm}$ during ablation. This technique has been recently introduced in the case of bulk targets and fullerene powders and has shown to be advantageous compared with static targets [31,32]. We compared HHG in nanoparticle-based plasmas and in plasmas generated by laser ablation of bulk targets of the same metallic elements (i.e., $\mathrm{Ag}, \mathrm{Al}$, and $\mathrm{Cu}$ ). Characterization of the plasma debris collected on the silicon substrates was carried out by scanning electron microscopy (SEM) and transmission electron microscopy (TEM).

\section{RESULTS AND DISCUSSION}

\section{A. Characterization of ablation deposits}

The as-supplied nanoparticles were analyzed by TEM. Figure 2 shows images in the case of silver and aluminum. The mean sizes of the corresponding individual nanoparticles were 25 and $20 \mathrm{~nm}$ respectively, in agreement with the specifications from the manufacturer. Notice that the original nanoparticles 


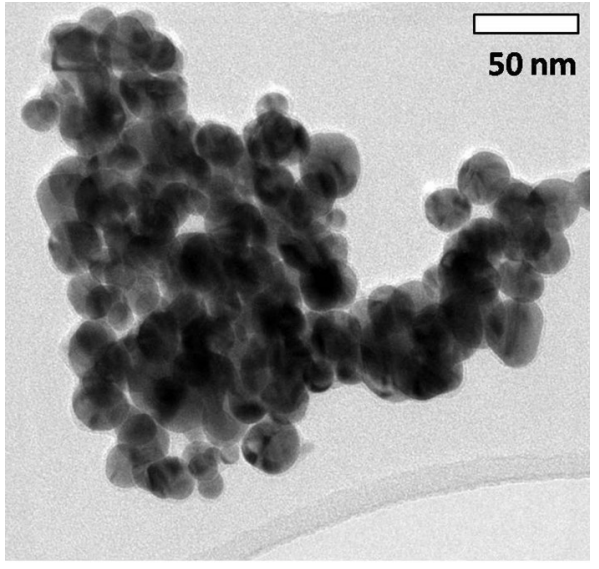

(a)

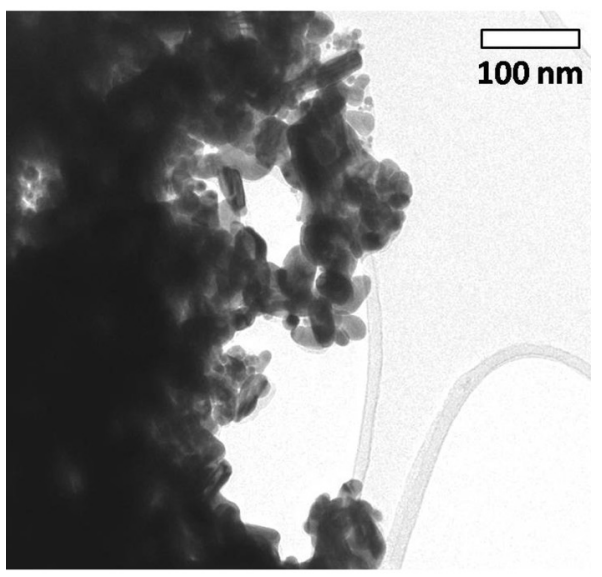

(b)

FIG. 2. TEM images of as-supplied (a) silver and (b) aluminum nanoparticles.

were combined in aggregates, containing thousands of separate clusters, as also shown in the SEM images displayed in Fig. 3.

To compare the dependence of the properties of the deposits with the high-order nonlinear optical response of the lasergenerated plasma under different ablating conditions, the nanoparticle-coated rotating rods were ablated using 160-ps pulses of the Ti:sapphire laser at $1 \mathrm{kHz}$, as well as $10-\mathrm{ns}$ pulses from a $\mathrm{Nd}$ :YAG laser operating at a repetition rate of $10 \mathrm{~Hz}$. Figure 4 shows the SEM pictures of the material ejected upon ablation of $\mathrm{Ag}, \mathrm{Al}$, and $\mathrm{Cu}$ nanoparticles using 160 -ps and 10-ns pulses. One can see that the characteristic sizes of the deposited nanostructured layer resemble those of the initial pristine nanoparticle targets (Fig. 3). Notice that we used moderate laser ablation intensities $\left(1 \times 10^{10}\right.$ and $3 \times 10^{9} \mathrm{~W} \mathrm{~cm}^{-2}$ for 160 -ps and 10 -ns pulses, respectively), corresponding in each case to conditions of optimal HG. Overexcitation of the nanoparticle-containing targets, by using higher fluences, did not yield nanostructured deposits as did those appearing in the images of Fig. 4, but rather a layer of chaotically appearing aggregates. Also, at high ablation fluences, the HHG yield decreased considerably or completely disappeared after a few thousand ablation pulses (i.e., after a few seconds of target irradiation), even when using the rotating target.

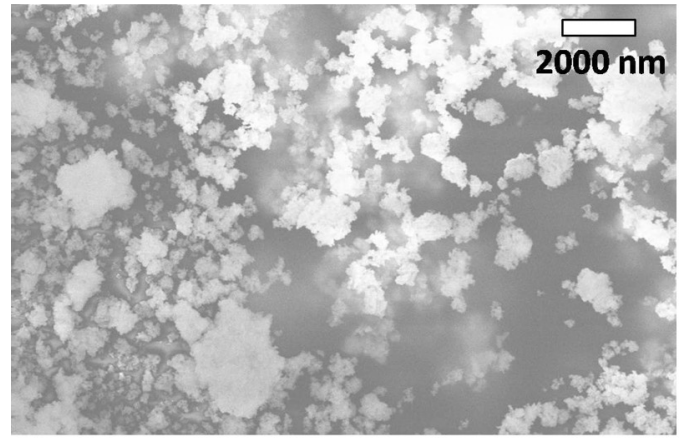

(a)

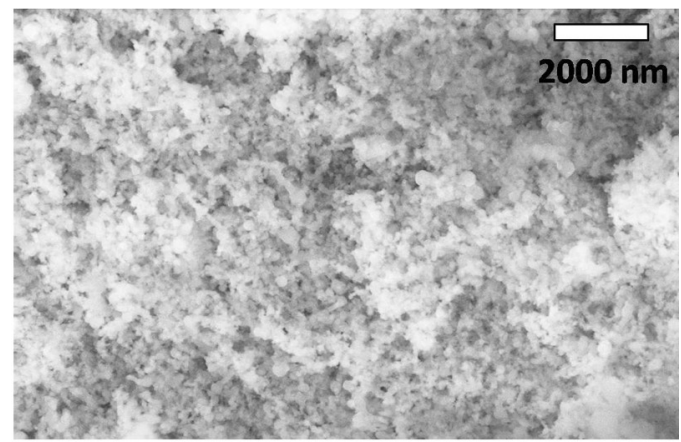

(b)

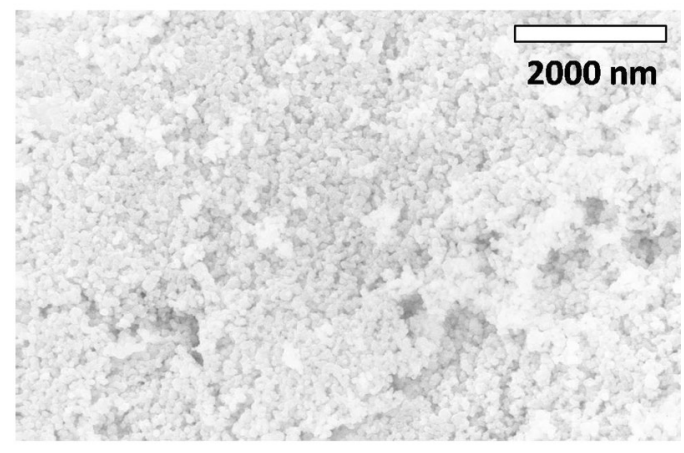

(c)

FIG. 3. SEM images of (a) silver, (b) aluminum, and (c) copper aggregates of nanoparticles before ablation.

We also analyzed the deposits generated upon laser ablation of the bulk metals $(\mathrm{Ag}, \mathrm{Al}$, and $\mathrm{Cu}$ ) at the ablation conditions that ensured optimum HHG conversion efficiency in the corresponding ablation plumes. Under these conditions, and contrary to the observation in the case of nanoparticle-based targets, the collected deposits did not show any evidence of nanoparticles and mostly present a flat surface, indicative of a layer created by atomic species (neutrals and ions) evaporated from the target and deposited on the substrate. For ablation with 160-ps pulses, only when the ablation intensity was substantially increased $\left(>3 \times 10^{10} \mathrm{~W} \mathrm{~cm}^{-2}\right)$ did nanoparticles appear on the $\mathrm{Si}$ substrate, as it is displayed in Figs. 5(a), 5(c), and 5(d) for the different bulk metallic targets. For ablation with 10-ns pulses, the deposits appear as flat, smooth layers on the Si substrate [Fig. 5(b)] in the entire fluence range. It is important to note that the high-fluence conditions that ensured deposition of nanoparticles from bulk 


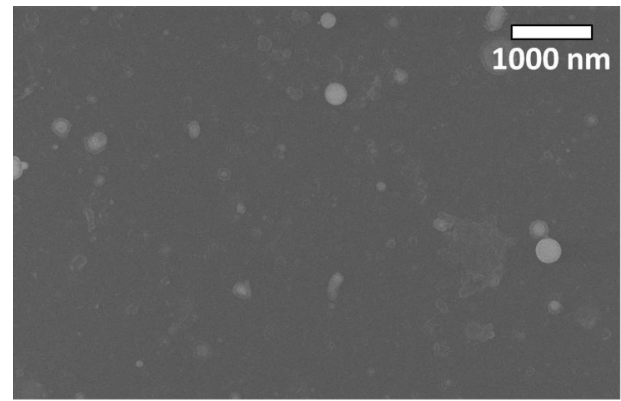

(a)

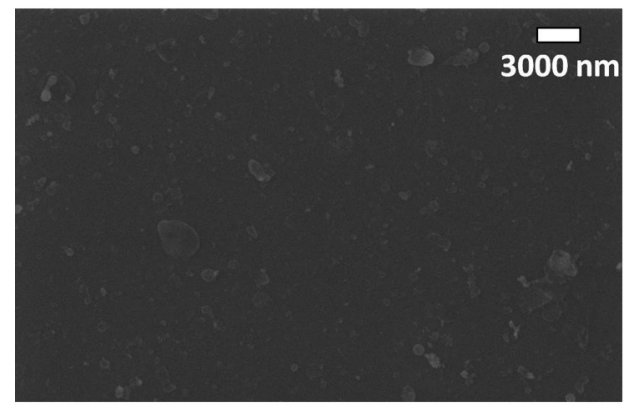

(c)

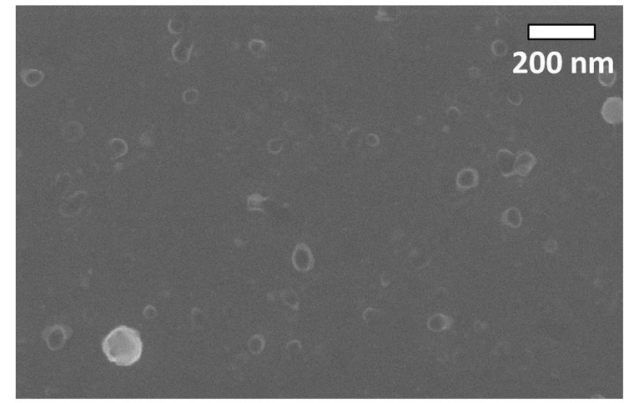

(e)

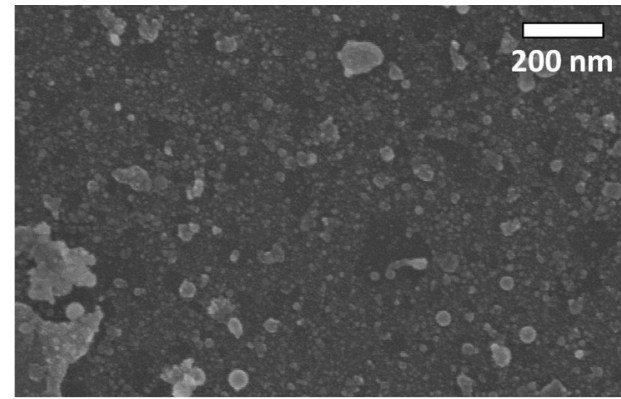

(b)

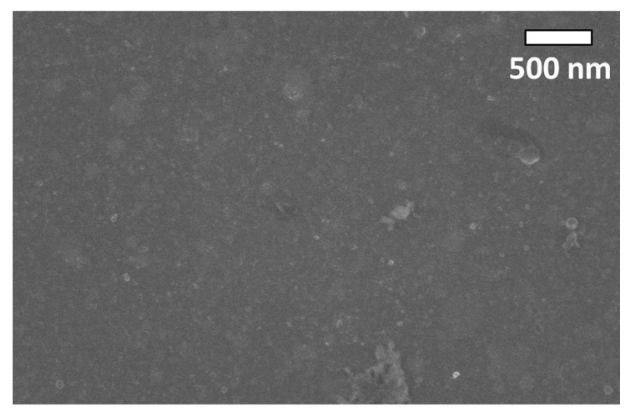

(d)

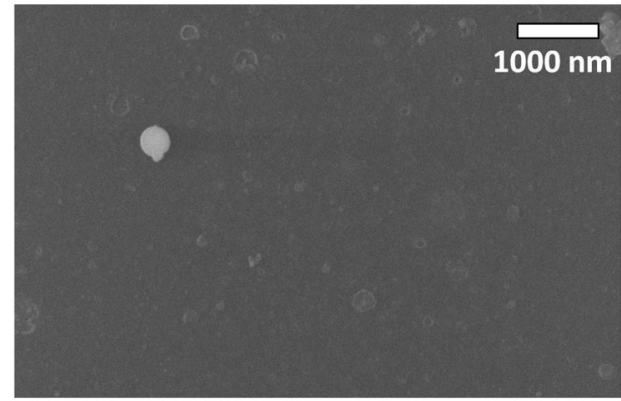

(f)

FIG. 4. SEM images of the material deposited on Si substrates by ablation of targets containing (a, b) Ag, (c, d) Al, and (e, f) Cu nanoparticles ablated with (a, c, e) 160-ps (1 kHz) and (b, d, f) 10-ns (10 Hz) pulses. The collection time for deposition was 3 minutes in both cases.

targets resulted in extremely inefficient HHG. Production of nanoparticles by laser ablation of metallic targets is a wellstudied phenomenon (see, for example, [33,34]). However, the use of high ablation fluences results in the generation of large free-electron densities, which is a detrimental factor in HHG due to the electron contribution to the phase vector mismatch between the driving and harmonic waves. This explains why, under ablation conditions leading to nanoparticle production in the plume of bulk metallic targets, the HHG signals are extremely weak; in this case, the presence of nanoparticles in the plasma does not compensate for the deteriorated phase mismatch conditions caused by overionization and production of large electron densities. Notice another extreme case when, by ablation of silver at an intensity of $10^{13} \mathrm{~W} \mathrm{~cm}^{-2}$, HG has previously been attributed to newly generated Ag nanoparticles [35].

The morphological characterization of ablation deposits was complemented by measurements of their absorption spectra. These measurements, performed using the plasma plume debris collected on glass substrates, are illustrated in
Fig. 6 for the case of silver. Figure 6(a) shows the appearance of a strong absorption band in the vicinity of the 400-500 nm region for deposits obtained by ablation of 25-nm silver nanoparticles using 160-ps and 10-ns pulses. The absorption band in each case is ultimately associated with the SPR of this metal and its position and width are related with the shape, size, structure, and assembly of the nanoparticles [36]. In fact, for this metal, one can note that the SPR band appearing on the deposits obtained by ablation with 160-ps pulses is related with the presence of small-sized silver clusters $(3-5 \mathrm{~nm})$. The TEM images of the as-supplied nanoparticle powder shown in Fig. 2 indicate the presence of nanoparticles with smaller sizes than the nominal $25 \mathrm{~nm}$. Thus the SPR band of these deposits reveals both the presence of nanoparticles directly ejected from the target and of those generated by disintegration of larger ones. The shape of the SPR band for deposits produced by ablation with $10-n$ s pulses is broader and redshifted with respect to that of the 160-ps pulse-induced deposits, indicating the larger characteristic sizes of deposits grown with ablation using longer laser pulses. 


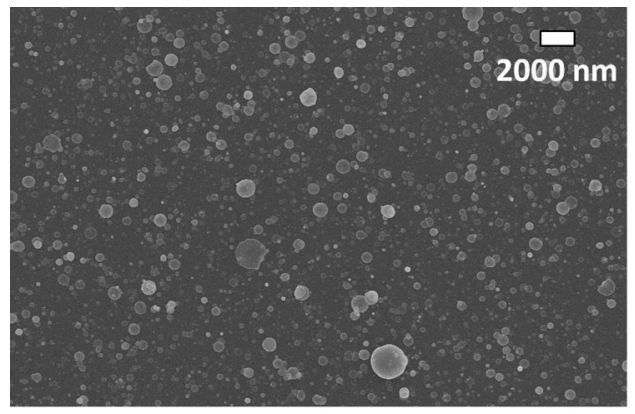

(a)

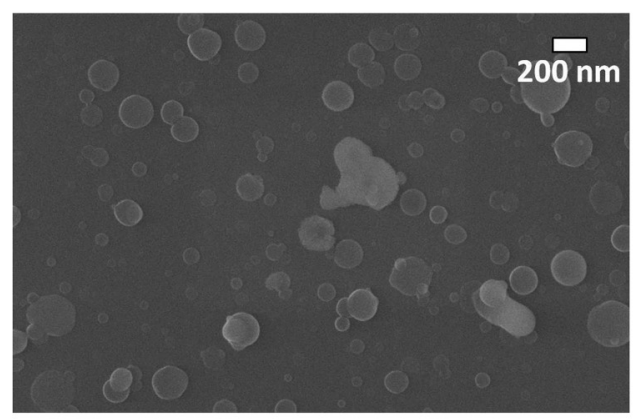

(c)

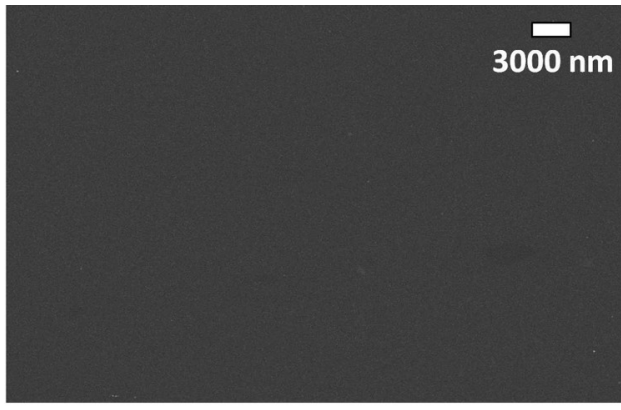

(b)

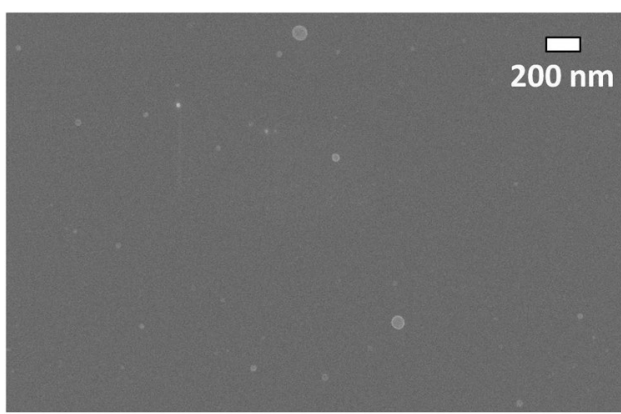

(d)

FIG. 5. SEM images of material deposited on Si substrates from ablation at high laser intensity of targets constituted by bulk (a, b) Ag, (c) $\mathrm{Al}$, and (d) $\mathrm{Cu}$ ablated with (a, c, d) 160-ps and (b) 10-ns pulses. The debris was deposited during 2 minutes.

In the spectral region explored, above $350 \mathrm{~nm}$, the deposits corresponding to ablation of Al nanoparticles did not show any characteristic absorption feature. This is most probably related with the sizes of the deposited nanostructures that, for values below $\approx 50 \mathrm{~nm}$, should present a SPR band at shorter wavelengths $(<300 \mathrm{~nm}$ [37]) than those explored. For the $\mathrm{Cu}$-nanoparticle-based target, absorption spectra of deposits (not shown) also displayed a band in the vicinity of $600 \mathrm{~nm}$, which can be assigned to the SPR of this metal [38]. However, this band is less defined as compared with the case of silver.

Absorption measurements of nanoparticle deposits originated from bulk silver upon ablation with 160-ps pulses, at the same high-fluence conditions as in Fig. 5(a), also show a strong absorption band, in this case centered at around $475 \mathrm{~nm}$ [Fig. 6(b), thick curve]. This is in sharp contrast with the absence of any particular spectral feature on the deposits collected by ablation with 10-ns pulses [Fig. 6(b), thin curve]. This difference can be understood in reference to Figs. 5(a) and 5(b), which show flat deposits for 10-ns ablation. This morphology is indicative of a highly atomized plume, mostly constituted by atoms and ions, which, once impinging on the substrate, produce a non-nanostructured metallic layer. It should be stressed that these ablation conditions did not lead to a measurable HHG signal.

\section{B. Harmonic generation from nanoparticle-containing plasmas}

Recently, HHG in laser plasmas generated from powderlike targets has been demonstrated using $\mathrm{C}_{60}$ and a high-pulserepetition laser [32]. In the present study, we extend this application to nanomaterials of considerably larger sizes with a $1-\mathrm{kHz}$ driving laser. The use of relatively large nanoparticles (of $\sim 20 \mathrm{~nm}$ in diameter) is expected to enhance the HHG yield, in reference to the small 0.7-nm fullerenes, due to the higher concentration of harmonic emitters [11]. The present experiments also provide the opportunity to compare HHG from single particles generated by ablation of bulk metal targets (atoms and ions) with clusters and agglomerates of atoms of the same element, entrained in the plume upon ablation of nanoparticle-based targets. Finally, we can study the effect of the nanoparticle size $(\sim 20 \mathrm{~nm}$ vs $\sim 100-150 \mathrm{~nm}$ ) on the nonlinear optical response of the plasma. The use of a high-repetition-rate driving laser $(1 \mathrm{kHz})$ is advantageous for the above studies as, by increasing the average power of the generated harmonics, less time is required for collecting all necessary data for a comparison of the highorder nonlinear optical properties of different plasmas. The application of $10-\mathrm{ns}, 10-\mathrm{Hz}$ heating pulses have also demonstrated harmonic generation from nanoparticle-containing plasmas; however, since our task was to analyze HHG from a high-pulse-repetition-rate laser, in what follows we concentrate on the results obtained using 160-ps, 1-kHz heating pulses.

Figure 7 shows a comparison of the harmonics generated between 40 and $65 \mathrm{~nm}$ from plasmas of a 25-nm silvernanoparticle-based target and from bulk silver upon the same 160-ps-pulse-induced ablation conditions (distance between the target surface and driving beam, ablation pulse duration, delay between ablation and driving pulses, etc.). Figure 7(a) shows CCD images of the harmonics, while Fig. 7(b) presents the relative intensity of the corresponding harmonic signals, which is in the range of 5-7 times higher for harmonics generated from nanoparticle-based targets compared with the HHG from ablation of bulk targets. The morphological and 


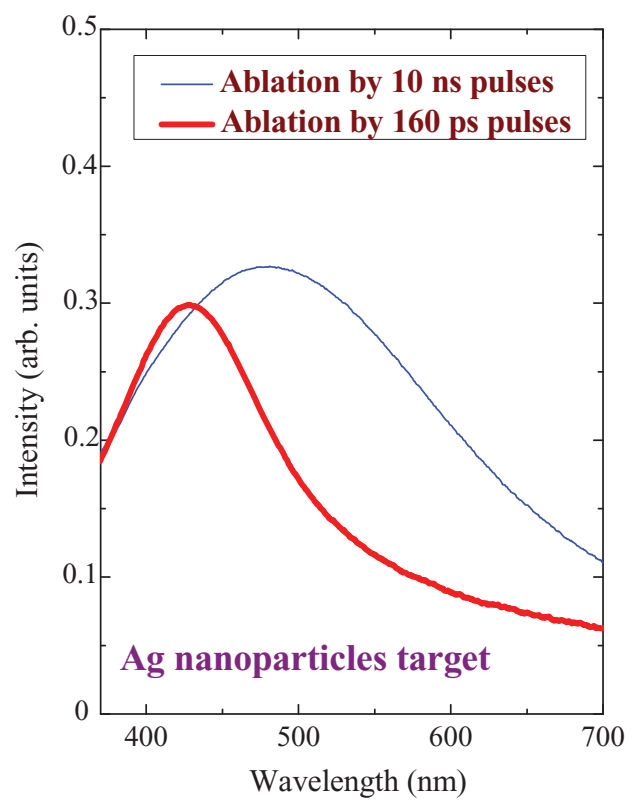

(a)

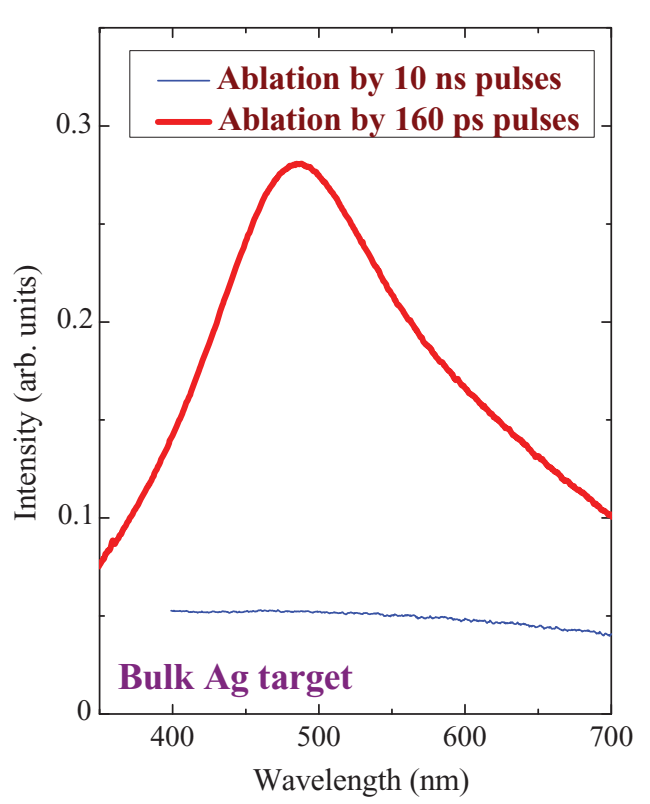

(b)

FIG. 6. (Color online) Absorption spectra of deposits generated from (a) an Ag nanoparticle-containing target and (b) bulk Ag for ablation by 160-ps (thick curves) and 10-ns (thin curves) pulses.

spectroscopic characterization of the deposits, presented in Sec. III A, indicates that by ablating with 160 -ps pulses, the former targets give rise to a plume mostly constituted by nanoparticles, while the plume of the bulk metal, at equal experimental conditions, is mostly populated with atomic species. It is also seen that the divergence and spectral width of the harmonics in the nanoparticle plumes are considerably larger than those of the atomic plumes. The image of nanoparticle-induced harmonics also displays spectral lines of ions generated by laser ablation of the metallic nanoparticles [Fig. 7(a), upper panel]. One can note that ionic lines in these spectra are related to the presence of a relatively large amount of free electrons in the plasma plume.

The role of electrons in deteriorating the conditions of $\mathrm{HHG}$ in gaseous media and plasmas has been investigated before. It has been reported [39] that strong harmonic emission from plasmas, mostly constituted by atomic species, was obtained in absence of the xuv spectra of plasma lines from excited ions. The simultaneous appearance of strong harmonics and ion lines points toward the growing influence of large clusters for creating favorable conditions for $\mathrm{HHG}$, even in the presence of some amount of free electrons. The prevailing influence of nanoparticles over free electrons in HHG, attributed to the increased cross section of recombination of the accelerated electron and to local field enhancement effects related with their plasmonic properties, is an interesting peculiarity of this process which needs further investigation.

For Al targets, the ratio of intensities between the harmonics generated from 20-nm nanoparticle-based targets and bulk metal targets was larger than from $\mathrm{Ag}$ and measured in the 7-11 range (Fig. 8). As in the case of $\mathrm{Ag}$, the former targets produce harmonics with larger divergence and spectral width at equal experimental conditions. In the case of the Al nanoparticle plasma, contrary to the case of Ag, we did not detect ion spectral lines alongside HG. The absence of ion lines signposts the lesser degree of ionization, and thus the lower free-electron density in the Al-based plumes, also partly explaining the better enhancement ratio, as compared with $\mathrm{Ag}$, of the nanoparticle-based target with respect to the bulk metal. Notice that we optimized the HHG from nanoparticles for both $\mathrm{Ag}$ and $\mathrm{Al}$ clusters. The excitations of cluster-containing targets at these conditions were different. Particularly, for Ag clusters, we used higher heating pulse intensity to achieve maximum conversion efficiency, compared with the case of aluminum nanoparticles. This difference in excitation at optimal conditions, when the strongest harmonics were achieved in both cases, led to appearance of ionic emission from silver nanoparticle plasma, while no analogous emission was registered from the plasma plumes containing Al clusters. Another reason for observing a better ratio in the case of aluminum species could be related with the higher efficiency of HHG from the plasmas created on the Ag bulk targets compared with the Al bulk targets.

Copper nanoparticles showed low HHG efficiency, possibly caused by less favorable plasma formation conditions involving aggregation into large nanoparticles during laser ablation. It should be mentioned that ablation of the glue used to coat the rotating rods with nanoparticle powders did not yield either HG nor atomic plasma emissions at the various experimental conditions investigated, thus confirming that the species responsible for harmonic generation are solely associated with the metallic samples.

The conditions of harmonics registration presented in Figs. 7 and 8 were different for the $\mathrm{Ag}$ and $\mathrm{Al}$ nanoparticles cases, since they were optimized with regard to the bulk species. The difference was particularly related to the collection time by the CCD camera. Figure 9 shows the relative harmonics from Ag- and Al-nanoparticle-containing 
13
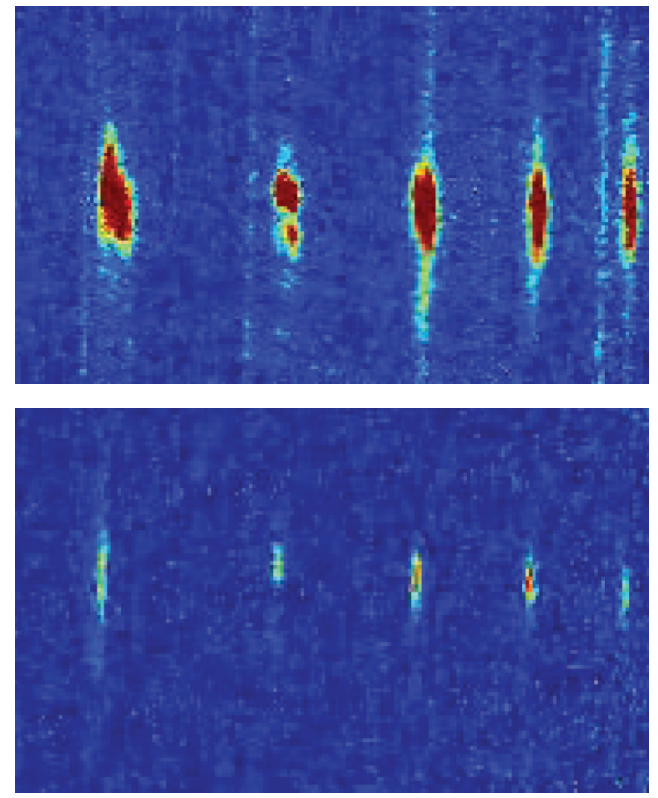

(a)

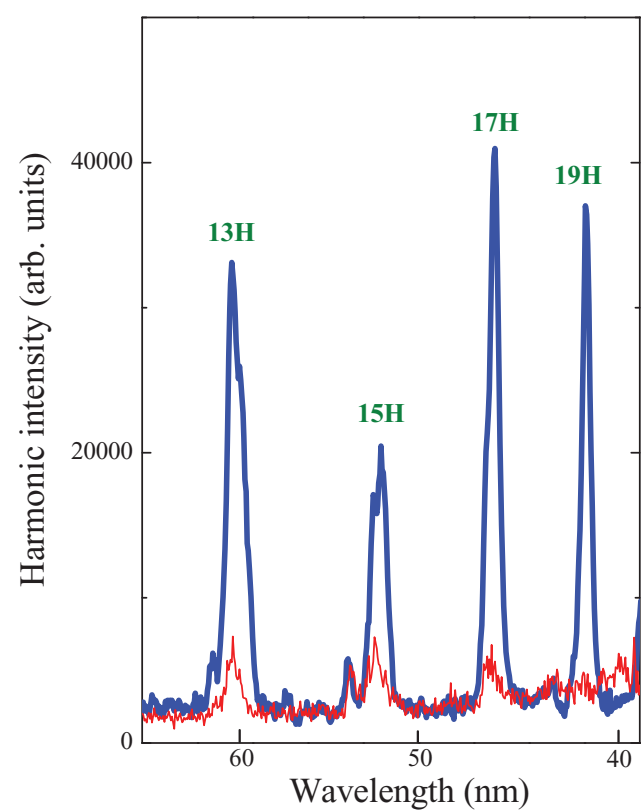

(b)

FIG. 7. (Color online) (a) Raw images of harmonic spectra in a laser plasma produced by ablation with 160-ps pulses of 25-nm Agnanoparticle-based (upper panel) and bulk Ag (bottom panel) targets. (b) Relative intensities of the harmonics generating in the silver nanoparticle (thick curve) and atomized (thin curve) plumes.

plasma plumes at equal conditions of registration, which is useful for direct comparison of the harmonic yields from these media.
The larger divergence of the harmonics generated from nanoparticle-based targets, as observed for Ag and Al, could be attributed to the stronger influence in the overall yield

\section{$\begin{array}{llllll}11 & 13 & 15 & 17 & 19 & 21\end{array}$}
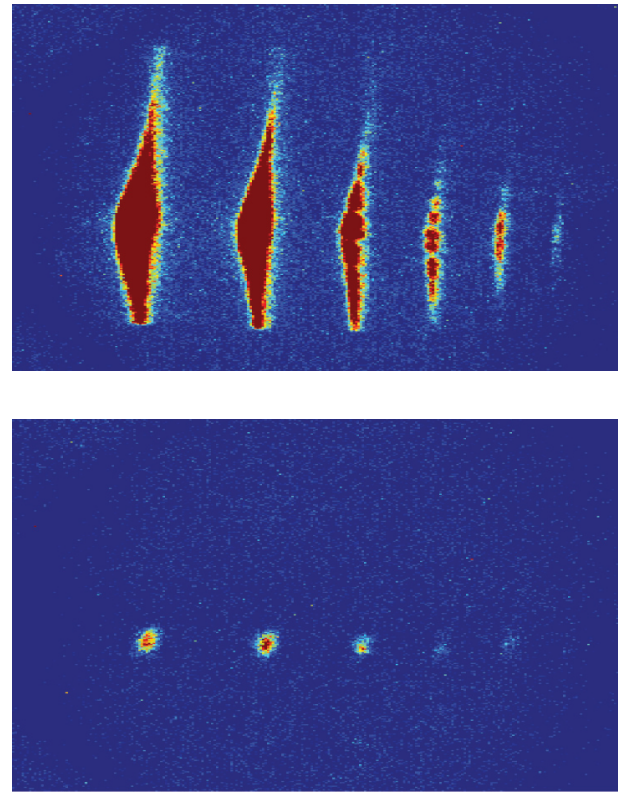

(a)

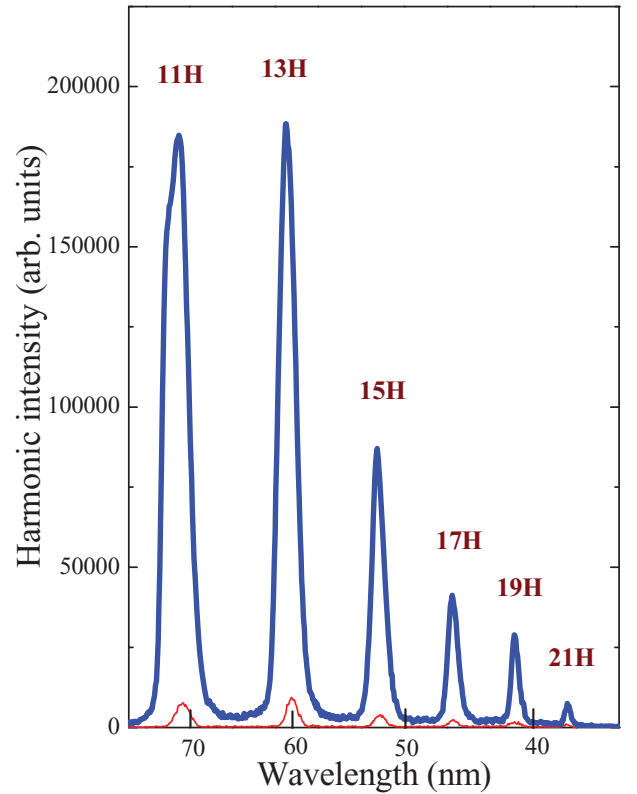

(b)

FIG. 8. (Color online) (a) Raw images of harmonic spectra in plasmas produced by ablation of 20-nm Al nanoparticles (upper panel) and bulk Al (bottom panel) targets. (b) Relative intensities of the harmonics generating in the aluminum nanoparticle (thick curve) and atomized (thin curve) plumes. 


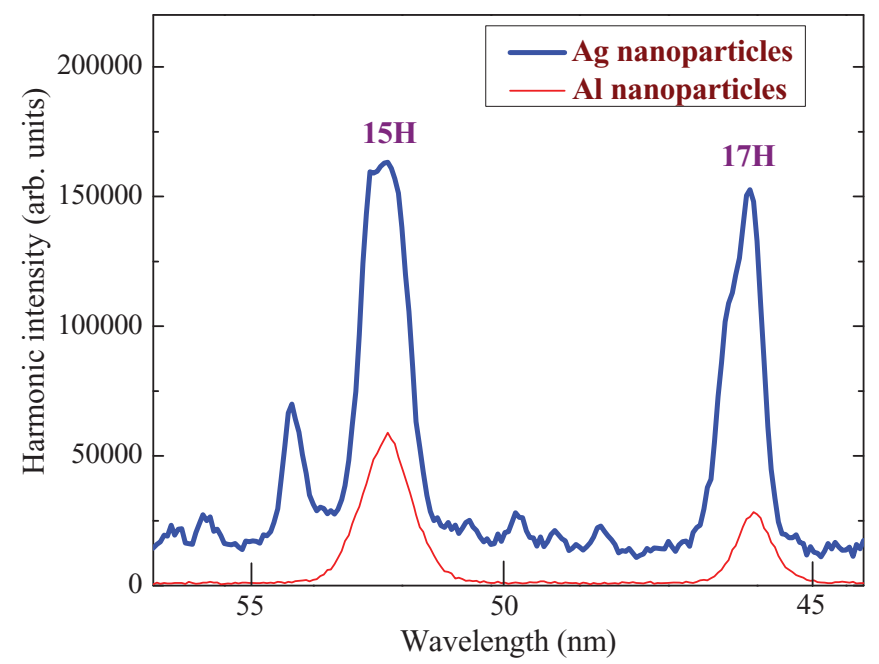

FIG. 9. (Color online) Comparative intensities of the 15th and 17th harmonics generated in the plasma plumes containing $\mathrm{Ag}$ (thick curve) and $\mathrm{Al}$ (thin curve) 20-25 nm sized nanoparticles.

of $\mathrm{HG}$ of the long trajectories of the accelerated electrons. This assumption is related to the increased recombination cross section of the returning electron moving along the long trajectory with larger particles and subsequent increased emission of harmonic photons.

We only observed relatively low-order harmonics from Agand Al-nanoparticle-containing plasmas (in the range of the 21 st and 27th harmonics) as compared with plasmas constituted by metallic atomic species (41st and 59th harmonics from $\mathrm{Al}$ and $\mathrm{Ag}$ targets, respectively). This is possibly because, in the former case, neutral metallic atoms within the plasma nanoparticles, with their relatively low ionization potential, are the main nonlinear species contributing to HG. In the meantime, in plasmas generated from bulk metallic targets, the contribution from atomic ions is predominant, leading to the extension of the harmonic cutoff.

The absolute value of conversion efficiency for the harmonics generating in the plateau region in the case of ablation of a bulk silver was estimated to be $\sim 10^{-5}$. An analogous value of conversion efficiency at approximately the same conditions was reported in [40], where the absolute measurements of harmonic efficiency from silver-containing plasma were carried out. From these estimates and relative intensities of harmonics from the plasmas produced on the bulk and cluster samples, the conversion efficiencies from small Ag- and Al-nanoparticle-containing plasmas were defined to be $6 \times 10^{-5}$ and $2 \times 10^{-5}$, respectively. These estimates show that, in the case of silver nanoparticle plasma, the maximum energy of each harmonic pulse in the range of the 13th to $21 \mathrm{st}$ orders was $6 \times 10^{-8} \mathrm{~J}$. Correspondingly, the average power of those harmonics at a $1-\mathrm{kHz}$ pulse repetition rate was $60 \mu \mathrm{W}$.

We also investigated, under the present experimental conditions, the process of $\mathrm{HHG}$ in plasmas generated from 100 to $150 \mathrm{~nm} \mathrm{Ag}$ and from 100-nm Al nanoparticles. The enhancement of the harmonic signal from these large nanoparticles with respect to that of the bulk target was found negligible. Ablation of targets constituted by larger nanoparticles most probably leads to the entrainment of larger clusters in the plume. This causes the reduction of the local field effect and contributes to a larger absorption probability of the generated short-wavelength radiation, explaining the observed effect. The optimal sizes of the nanoparticles participating in $\mathrm{HG}$ is an unresolved issue that should be investigated further due to the necessity of defining the relative role of the different competitive mechanisms of HHG in clustered plasmas.

In all, the comparative measurements of $\mathrm{HG}$ from three different plasmas containing $\mathrm{Ag}, \mathrm{Al}$, and $\mathrm{Cu}$ nanoparticles of approximately the same size $(\sim 20-25 \mathrm{~nm})$ have shown that Ag nanoparticles provide the highest conversion efficiency into the xuv range. The superior performance of silver could be attributed to the influence of the SPR of this metal. The wavelength of the second harmonic of the driving field $(390 \mathrm{~nm})$ almost coincides with the short-wavelength wing of the broad SPR band of the Ag nanoparticle emitters (see Fig. 6). This effect is foreseen to enhance the nonlinear susceptibility of the medium and favor the HHG yield. Although it is still unclear how the size of the plasma nanoparticles influences the enhancement of harmonic yield, it is expected that the increase of nanoparticle size would lead to an increase of the recombination cross section with the returning electron. However, as mentioned before, HG generation in clusters containing a large number of atomic particles can be restricted by atomic absorption of the generated harmonic waves.

The uncertainty of the exact mechanism of HHG from clusters has previously been outlined in a few papers [20,41-43]. Among the considered channels, ionization and recombination to the same atom, to the neighboring ions, and to the whole nanoparticle have been proposed. In particular, Ruf et al. [20] attributed $\mathrm{HHG}$ in $\mathrm{Kr}$ clusters to a mechanism in which delocalized electrons tunnel form a cluster and recollide coherently with the whole cluster. Notice that the experiments with gas clusters have revealed a difficulty in disentangling the harmonics produced by different species (monomers and clusters of different sizes). In the meantime, our comparative studies of HHG in plasmas constituted by clusters and monomers show that, at equal experimental conditions, the latter emitters provide considerably weaker harmonic yield, thus pointing to the specific advanced properties of the clustered emitters of harmonics in the nanoparticle-containing plasmas.

\section{CONCLUSIONS}

In conclusion, we have measured HHG using a $1-\mathrm{kHz}$, Ti:sapphire driving laser in plasma plumes containing $\mathrm{Ag}$, $\mathrm{Al}$, and $\mathrm{Cu}$ metal nanoparticles and have demonstrated that some of these plasma species allow the efficient conversion of the IR photons towards the short-wavelength range. This was made possible by using rotating rods targets that produced plasmas of relatively high stability under high-repetition-rate ablation. Analyses of the morphology and the absorption spectra of the ablation deposits has allowed the definition of conditions for generating plasmas containing nanoparticles of the same characteristics as the ones used as target material. We have discussed the relation between the properties of the deposits and the characteristics of the HHG spectra and the conversion efficiency. Plasmas mainly constituted by metallic clusters show higher conversion efficiency than plasmas mostly containing metallic atomic species. Some 
issues should be addressed to promote the robust use of this nanoparticle-containing media for generation of coherent xuv and soft $\mathrm{x}$-ray radiation, mainly related with the long-range stability of the generated harmonics. However, the results herein have shown the good prospects of the method for studying the high-order optical nonlinearities of powdered media, especially those with nanoscale sizes.

\section{ACKNOWLEDGMENTS}

These studies were supported by the EPSRC program (Grants No. EP/F034601/1, No. EP/E028063/1, and No.
EP/I032517/1). R.A.G. acknowledges support from a Marie Curie International Incoming Fellowship Grant within the $7^{\text {th }}$ European Community Framework Program (Grant No. PIIF-GA-2009-253104). I.L.Q. is thankful for support from an FPI fellowship (MINECO, Spain). M.C. is grateful for support from the Programa Nacional de Movilidad de Recursos Humanos, Plan Nacional de I-D + i 2008-2011, Spain. D.Y.L. acknowledges 1-ZVAL and 1-ZVAW grants administrated by The Hong Kong Polytechnic University. The authors thank S. A. Maier and T. Roschuk for their support of the morphological studies of samples.
[1] A. McPherson, G. Ginson, H. Jara, N. Johann, I. A. McIntyre, K. Boyer, and C. K. Rhodes, J. Opt. Soc. Am. B 4, 595 (1987).

[2] M. Ferray, A. L'Huillier, X. F. Li, L. A. Lompré, G. Mainfray, and G. Manus, J. Phys. B: At. Mol. Opt. Phys. 21, L31 (1988).

[3] L. A. Lompré, A. L'Huillier, M. Ferray, P. Monot, G. Mainfray, and G. Manus, J. Opt. Soc. Am. B 7, 754 (1990).

[4] Y. Akiyama, K. Midorikawa, Y. Matsunawa, Y. Nagata, M. Obara, H. Tashiro, and K. Toyoda, Phys. Rev. Lett. 69, 2176 (1992).

[5] P. A. Franken, A. E. Hill, C. W. Peters, and G. Weinreich, Phys. Rev. Lett. 7, 118 (1961).

[6] K. Miyazaki and H. Takada, Phys. Rev. A 52, 3007 (1995).

[7] I. P. Christov, M. M. Murnane, and H. C. Kapteyn, Phys. Rev. Lett. 78, 1251 (1997).

[8] G. Tempea, M. Geissler, M. Schnürer, and T. Brabec, Phys. Rev. Lett. 84, 4329 (2000).

[9] A. Pirri, C. Corsi, and M. Bellini, Phys. Rev. A 78, 011801 (2008).

[10] J.-F. Hergott, M. Kovacev, H. Merdji, C. Hubert, Y. Mairesse, E. Jean, P. Breger, P. Agostini, B. Carré, and P. Salières, Phys. Rev. A 66, 021801 (2002).

[11] J. F. Reintjes, Nonlinear Optical Parametric Processes in Liquids and Gases (Academic Press, Orlando, FL, 1984).

[12] R. A. Ganeev, M. Suzuki, T. Ozaki, M. Baba, and H. Kuroda, Opt. Lett. 31, 1699 (2006).

[13] R. A. Ganeev, M. Baba, M. Suzuki, and H. Kuroda, Phys. Lett. A 339, 103 (2005).

[14] T. D. Donnelly, T. Ditmire, K. Neuman, M. D. Perry, and R. W. Falcone, Phys. Rev. Lett. 76, 2472 (1996).

[15] J. W. G. Tisch, T. Ditmire, D. J. Fraser, N. Hay, M. B. Mason, E. Springate, J. P. Marangos, and M. H. R. Hutchinson, J. Phys. B: At. Mol. Opt. Phys. 30, L709 (1997).

[16] S. X. Hu and Z. Z. Xu, Appl. Phys. Lett. 71, 2605 (1997).

[17] J. W. G. Tisch, Phys. Rev. A 62, 041802(R) (2000).

[18] C. Vozzi, M. Nisoli, J.-P. Caumes, G. Sansone, S. Stagira, S. De Silvestri, M. Vecchiocattivi, D. Bassi, M. Pascolini, L. Poletto, P. Villoresi, and G. Tondello, Appl. Phys. Lett. 86, 111121 (2005).

[19] C.-H. Pai, C. C. Kuo, M.-W. Lin, J. Wang, S.-Y. Chen, and J.-Y. Lin, Opt. Lett. 31, 984 (2006).

[20] H. Ruf, C. Handschin, R. Cireasa, N. Thiré, A. Ferré, S. Petit, D. Descamps, E. Mével, E. Constant, V. Blanchet, B. Fabre, and Y. Mairesse, Phys. Rev. Lett. 110, 083902 (2013).
[21] C. Hubert, L. Billot, P.-M. Adam, R. Bachelot, P. Royer, J. Grand, D. Gindre, K. D. Dorkenoo, and A. Fort, Appl. Phys. Lett. 90, 181105 (2007).

[22] S. Kim, J. Jin, Y.-J. Kim, I.-Y. Park, Y. Kim, and S.-W. Kim, Nature (London) 453, 757 (2008).

[23] T. Hanke, G. Krauss, D. Traütlein, B. Wild, R. Bratschitsch, and A. Leitenstorfer, Phys. Rev. Lett. 103, 257404 (2009).

[24] K. D. Ko, A. Kumar, K. H. Fung, R. Ambekar, G. L. Liu, N. X. Fang, and K. C. Toussaint, Nano Lett. 11, 61 (2011).

[25] R. A. Ganeev, L. B. Elouga Bom, J. Abdul-Hadi, M. C. H. Wong, J. P. Brichta, V. R. Bhardwaj, and T. Ozaki, Phys. Rev. Lett. 102, 013903 (2009).

[26] R. A. Ganeev, L. B. Elouga Bom, M. C. H. Wong, J.-P. Brichta, V. R. Bhardwaj, P. V. Redkin, and T. Ozaki, Phys. Rev. A 80, 043808 (2009).

[27] R. A. Ganeev, M. Suzuki, M. Baba, M. Ichihara, and H. Kuroda, J. Appl. Phys. 103, 063102 (2008).

[28] R. A. Ganeev, L. B. Elouga Bom, and T. Ozaki, J. Phys. B: At. Mol. Opt. Phys. 42, 055402 (2009).

[29] H. Singhal, R. A. Ganeev, P. A. Naik, A. K. Srivastava, A. Singh, R. Chari, R. A. Khan, J. A. Chakera, and P. D. Gupta, J. Phys. B: At. Mol. Opt. Phys. 43, 025603 (2010).

[30] R. A. Ganeev, Laser Phys.18, 1009 (2008).

[31] C. Hutchison, R. A. Ganeev, T. Witting, F. Frank, W. A. Okell, J. W. G. Tisch, and J. P. Marangos, Opt. Lett. 37, 2064 (2012).

[32] R. A. Ganeev, C. Hutchison, T. Witting, F. Frank, S. Weber, W. A. Okell, E. Fiordilino, D. Cricchio, F. Persico, A. Zaïr, J. W. G. Tisch, and J. P. Marangos, J. Opt. Soc. Am. B 30, 7 (2013).

[33] T. Donnelly, J. G. Lunney, S. Amoruso, R. Bruzzese, X. Wang, and X. Ni, J. Appl. Phys. 108, 043309 (2010).

[34] M. López-Arias, M. Oujja, M. Sanz, R. A. Ganeev, G. S. Bolataev, N. K. Satlikov, R. I. Tugushev, T. Usmanov, and M. Castillejo, J. Appl. Phys. 111, 043111 (2012).

[35] H. Singhal, R. A. Ganeev, P. A. Naik, J. A. Chakera, U. Chakravarty, H. S. Vora, A. K. Srivastava, C. Mukherjee, C. P. Navathe, S. K. Deb, and P.D. Gupta, Phys. Rev. A 82, 043821 (2010).

[36] V. Amendola, O. M. Bakr, and F. Stellacci, Plasmonics 5, 85 (2010).

[37] Y. Ekinci, H. H. Solak, and J. F. Löffler, J. Appl. Phys. 104, 083107 (2008) 
[38] G. H. Chan, J. Zhao, E. M. Hicks, G. C. Schatz, and R. P. Van Duyne, Nano Lett. 7, 1947 (2007).

[39] R. A. Ganeev, J. Modern Opt. 59, 409 (2012).

[40] R. A. Ganeev, P. A. Naik, H. Singhal, J. A. Chakera, M. Kumar, U. Chakravarty, and P. D. Gupta, Opt. Commun. 285, 2934 (2012).
[41] P. Moreno, L. Plaja, and L. Roso, Europhys. Lett. 28, 629 (1994).

[42] V. Véniard, R. Taïeb, and A. Maquet, Phys. Rev. A 65, 013202 (2001).

[43] D. F. Zaretsky, P. Korneev, and W. Becker, J. Phys. B 43, 105402 (2010). 